\title{
Evaluation of anatomical obstruction by Doppler echocardiography and magnetic resonance imaging in patients with coarctation of the aorta
}

\author{
Dag E Teien, Hans Wendel, Jan Björnebrink, Leif Ekelund
}

\begin{abstract}
Objective-To evaluate a new Doppler echocardiographic index of obstruction in patients with native coarctation or recoarctation.
\end{abstract}

Patients-32 patients (mean age 20, range 3 months-50 years).

Methods-Magnetic resonance imaging (MRI) was used to investigate the descending aorta. The cross sectional area of the obstruction was compared with the area of the abdominal aorta as an index of obstruction (CoA index). Doppler echocardiography was used to record the velocities in the coarctation jet and in the abdominal aorta. According to the continuity equation the ratio of these velocities should equal the ratios of the cross sectional areas. The gradients and diastolic half time of the Doppler curve were calculated.

Results-There was a close correlation between the MRI index of obstruction and the Doppler velocity ratio $(r=0.92)$. The sensitivity and specificity of this ratio in detecting a CoA index equal to or less than 0.25 were better than those obtained with gradients only or the combination of gradients and diastolic half time.

Conclusion-The new Doppler velocity ratio correlated closely with an anatomical index of obstruction. It was easy to record in most patients and it avoided difficulties about the choice of instantaneous or peak to peak gradients and whether or not to substract proximal velocities for the calculation of gradients.

(Br Heart F 1993;69:352-355)

Coarctation of the aorta is characterised by increased proximal blood pressure and anatomical obstruction in the ascending aorta. It is difficult to evaluate this obstruction because of the variability in cardiac output, number and size of collaterals, and peripheral resistance. Nevertheless the pressure drop across the obstruction (the gradient) giving rise to an unknown flow through an unknown obstruction has been widely used to quantify the obstruction. ${ }^{12}$ The gradient can be measured invasively, by cuffs, or by Doppler echocardiography. In adults and in children with aortic valve stenosis the relation between gradients and obstruction is of limited usefulness ${ }^{34}$ as are gradients and 8 the pressure half time method in patients with mitral stenosis. ${ }^{5}$ We think it unlikely that a o single index of obstruction such as a gradient, 0 pressure half time, or diastolic flow will yield better results in patients with coarctation of $\rightarrow$ the aorta where flow/obstruction relations are even more complex than in valve lesions. Limited correlations have been reported $\&$ between obstruction and gradients at rest and during exercise and between obstruction and proximal blood pressure. ${ }^{6}$

When Doppler echocardiography is used to estimate the gradient across the coarctation $\stackrel{\circ}{\varnothing}$ site it is the peak instantaneous gradient that is estimated and this is conceptually different from the traditional peak to peak gradient $\frac{}{\sigma}$ and is always larger. ${ }^{7}$ Another source of confusion is the subtraction of proximal velocities when the Bernoulli transfer from velocities to gradients is used and whether proximal velocities in the descending aorta can be replaced 3 by velocities in the ascending aorta. We report a Doppler method that may give the $\frac{}{3}$ same anatomical information as angiography $O$ or magnetic resonance imaging (MRI). It gives an index of obstruction based on the relation between the cross sectional area of the obstruction and the cross sectional area of $\%$ the abdominal aorta.

Patients and methods

We studied 32 patients (19 men) (mean age $\stackrel{\overbrace{}}{\varnothing}$ 20 years, range 3 months- 50 years, median 33 years). Twenty seven patients had had $\frac{0}{0}$ operations and five had native coarctations. Three patients had mild aortic stenosis and $\frac{\rho}{\mathbb{Q}}$ four had mild to moderate aortic regurgita- $\frac{\varrho}{0}$ tion.

\section{METHOD}

\section{Magnetic resonance imaging}

Gated sagittal MR images of the thoracic aorta were obtained with a low field resistive magnet operating at $0.04 \mathrm{~T}$ (Magnaview, Instrumentarium, Finland). All 32 patients were studied in a close-coupled body coil with a spin echo (SE) pulse sequence (TR 


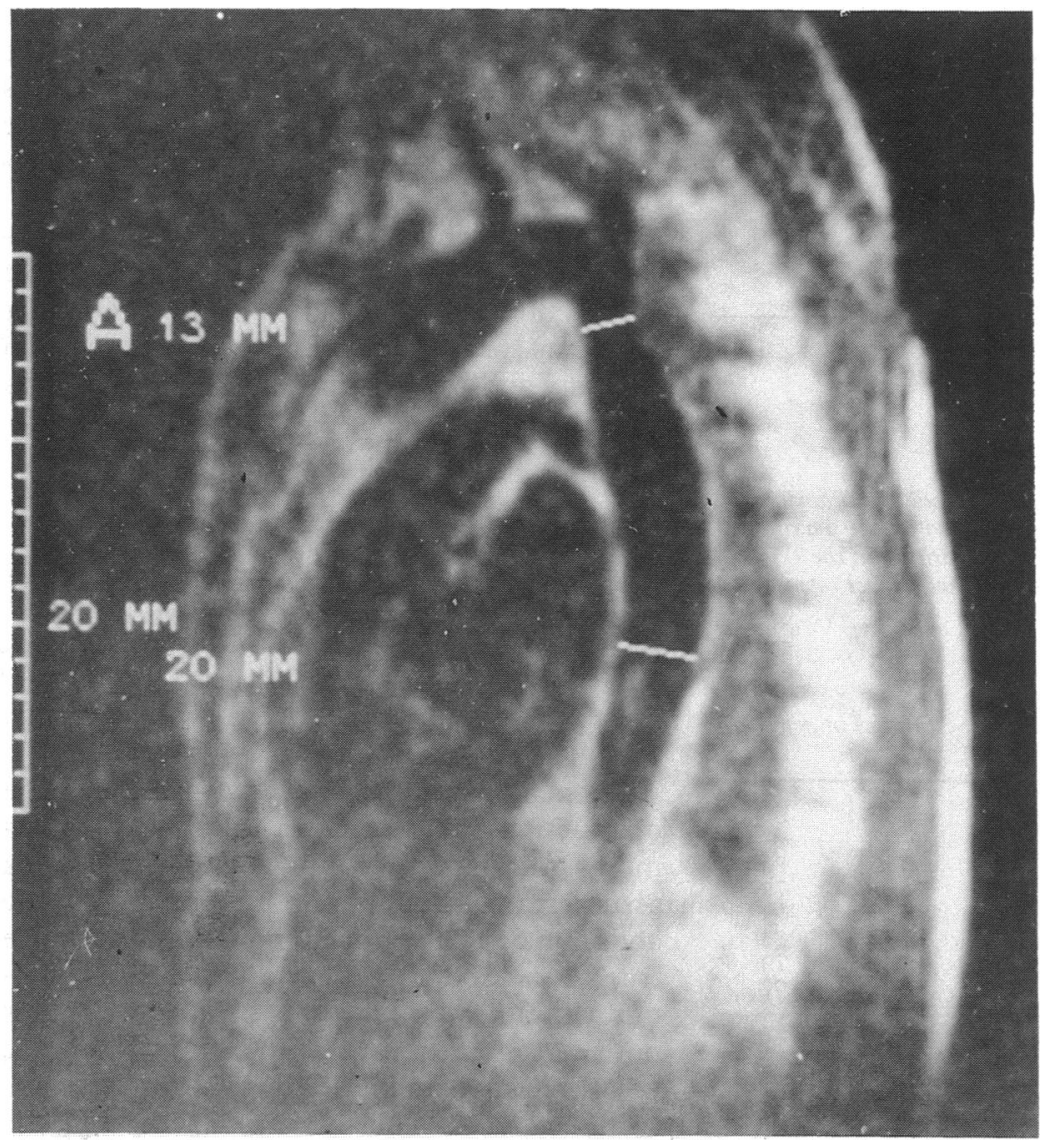

Figure 1 Magnetic resonance imaging of aorta.

$500 \mathrm{~ms}$, TE $40 \mathrm{~ms}, 3$ excitations, acquisition time 10-12 min depending on the pulse rate, giving 5 overlapping slices with a thickness of 7 or $10 \mathrm{~mm}$ ); field of view $31 \times 25 \mathrm{~cm}$, with a possible variation from $60-140 \%$ and a measuring matrix of $256 \times 256$ pixels. The Fredriksen's index of obstruction (CoA index $)^{8}$ was calculated as the ratio between the cross sectional area of the obstruction and the abdominal aorta somewhat above the level of the diaphragm. The diameters of the obstruction and the abdominal aorta were measured and the ratio of their squares calculated, assuming a circular geometry (fig 1).

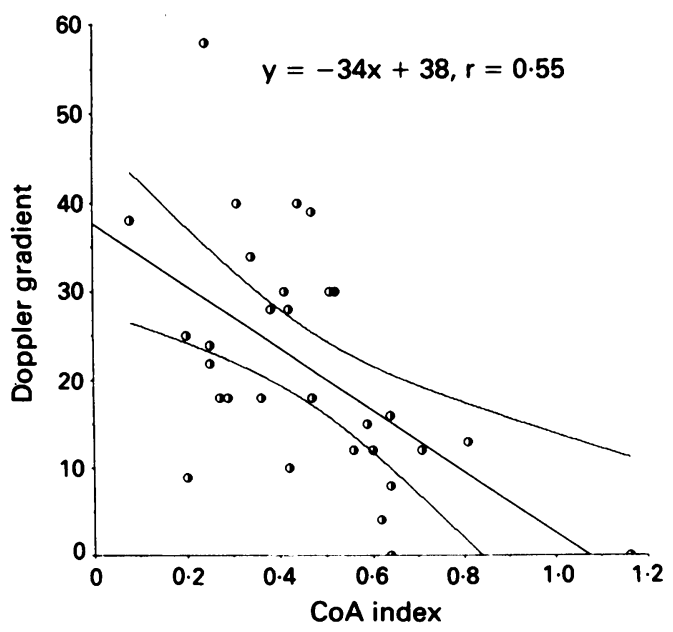

Figure 2 Doppler gradient plotted against the index of anatomical obstruction. $95 \%$ confidence intervals for the true mean of $y$ are shown. There are two overlapping points.

\section{Doppler echocardiography}

The continuity equation states that the products of the velocity and cross sectional area at two sites in a tubing system are equal. The ratio of velocities will then reflect the ratios of the cross sectional areas. Application of this equation to the descending aorta in a patient with coarctation assumes that the spatial velocity profiles at the level of the aortic obstruction and in the aorta at the diaphragmatic level are flat and that no major flow leaves or arrives at aorta between the two recording sites.

The patients were examined with Vingmed CF750 equipment. The maximal velocity in the coarctation jet (V1) was recorded by a single $\mathrm{MHz}$ continuous wave transducer. The probe was positioned in the supersternal notch and a good signal was achieved by angulating the probe. No angle corrections were done. From the subcostal position the abdominal aorta was found and a pulsed Doppler recording was made of the velocity in the abdominal aorta (V2). In the last 14 patients the colour flow map of the abdominal aortic flow was transformed to an in line Macintosh II computer for analysis of flow profile across the aortic lumen as well as angle corrections. In the remaining patients the angle corrections were made from the cross sectional picture. The ratio between the angle-corrected distal velocity (V2) and jet

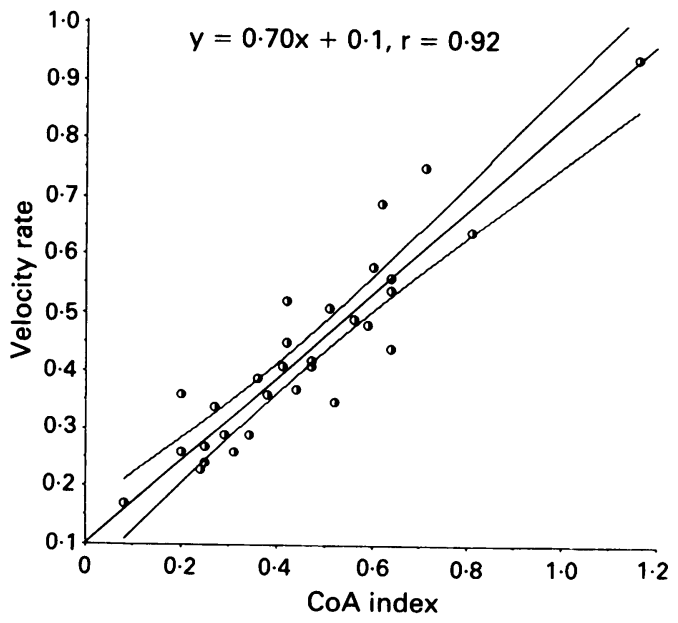

Figure 3 Velocity ratio plotted against the index of anatomical obstruction. $95 \%$ confidence intervals for the true mean of $y$ are shown. There are two overlapping points.

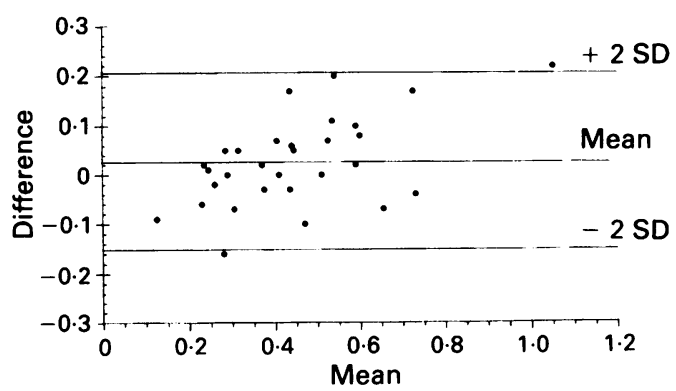

Figure 4 Difference between the non-invasively measured velocity ratio and the MRI CoA index plotted against the means of both methods. There are two overlapping points. 


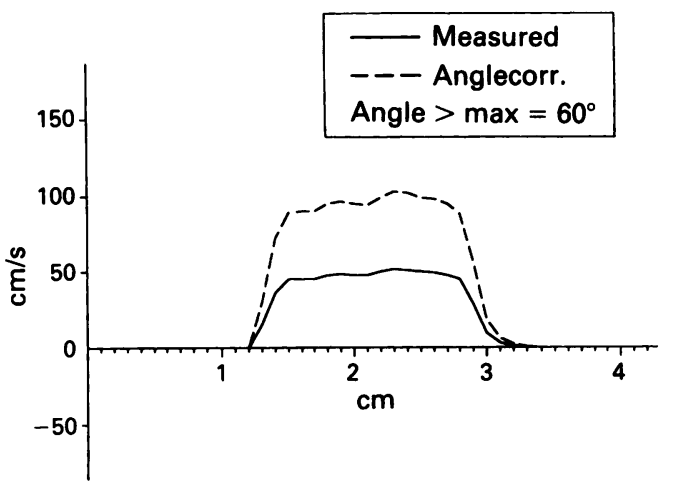

Figure 5 Velocity profile across the abdominal aorta in one patient. The blood velocity is plotted on the $y$ axis and the $x$ axis represents the anatomical position in the abdominal aorta. The highest of the two curves represents the angle corrected velocities.

Sensitivity and specificity of Doppler estimates in detecting a $\operatorname{Co} A$ index $<0 \cdot 25$

\begin{tabular}{lll}
\hline & $S e(\%)$ & $S p(\%)$ \\
\hline$P_{\max }>35$ (proximal velocity suntracted) & 43 & 83 \\
$P_{\max }>40$ (proximal velocity & 43 & 79 \\
$\quad$ not subtracted) & 71 & 83 \\
$P_{\max }>35$ or T $1 / 2$ PDV $>100 \mathrm{~ms}$ & 71 & 79 \\
$P_{\max }>40$ or $1 / 2$ PDV $>100 \mathrm{~ms}$ & 86 & 92 \\
Velocity ratio $<0.27$ &
\end{tabular}

PDV, peak diastolic velocity; $\mathrm{Q} S \mathrm{Se}(\%)$, sensitivity; $\mathrm{Sp}(\%)$, specificity.

velocity (V1) was calculated to give a noninvasive coarctation index of obstruction:

CoA index (non-inv) $=\mathrm{V} 2$ (angle corr) $/ \mathrm{V} 1$

In all patients the peak instantaneous gradients was calculated as: peak instantaneous gradient $=4 \times\left(\mathrm{V}_{\mathrm{J}}^{2} \times \mathrm{V}_{\mathrm{P}}^{2}\right)$, where $\mathrm{V}_{\mathrm{J}}=$ maximal jet velocity $(\mathrm{m} / \mathrm{s})$ and $V_{P}=$ velocity in the ascending aorta $(\mathrm{m} / \mathrm{s}) .{ }^{9}$ We measured the diastolic velocity half time ( $\mathrm{T} 1 / 2$ peak diastolic velocity (PDV)) in ms (the time for the diastolic velocity to fall to half its early value at the end of the $T$ wave $^{10}$ from the Doppler tracings.

STATISTICAL ANALYSIS

Means and standard deviations were calculated. Data were compared by linear regression analysis. Agreement between measurements was analysed according to the method of Bland and Altman. ${ }^{11}$

\section{Results}

The mean of the Doppler gradients was $23 \mathrm{~mm} \mathrm{Hg}$ (range $0-58 \mathrm{~mm} \mathrm{Hg}$ ), the mean of the Doppler estimated velocity ratios was 0.42 (range $0 \cdot 17-0.94$ ), and the mean of the MRI CoA indices was 0.45 (range $0 \cdot 08-1 \cdot 16)$. The diastolic velocity half time was more than $100 \mathrm{~ms}$ in three patients.

The correlation between peak instantaneous gradient and the MRI CoA index was 0.55 , SD $12 \mathrm{~mm} \mathrm{Hg}$ (fig 2). The correlation between the non-invasive $\mathrm{CoA}$ index and MRI CoA index was $0.92, \mathrm{SD}=0.07$ (fig 3). The difference between the non-invasive and invasive obstruction index was plotted against the means of both methods (fig 4). In only two patients was the difference slightly more than $2 \mathrm{SD}$. In the 14 patients in whom the flow profile across the aortic lumen was determined the mean difference between the maximal velocity and the spatial mean velocity was $0.14 \mathrm{~cm} / \mathrm{s}, S D=0.07 \mathrm{~cm} / \mathrm{s}$ (fig 5).

The table shows the sensitivity and specificity of Doppler gradients with and without $\stackrel{T}{\Phi}$ subtraction of proximal velocities, Doppler gradients or diastolic half time more than. $100 \mathrm{~ms}$, and the velocity ratio for detecting $\mathrm{a}=\overrightarrow{\mathrm{F}}$ CoA index of $<0 \cdot 25$.

\section{Discussion}

The results presented in this study indicate that the velocity ratio is better than gradients for the evaluation of anatomical obstruction. Several points must be considered.

\section{THE REFERENCE METHOD}

The choice of the best section to image the obstruction will always be difficult. Further- + more the use of the coarctation index assumes that both the obstruction and the ${ }_{\circ}$ abdominal aorta are circular. This may be true of the abdominal aorta but it is an obvi- $\rightarrow$ ous simplification for the obstruction. This potential error is further increased when the $\vec{\bullet}$ measured diameters are squared to give cross $c$ sectional areas. The thickness of the slices is not optimal.

\section{REGARDING THE VELOCITY RATIO}

For this new method it is assumed that no major flow arrives at or leaves the abdominal $\stackrel{\varrho}{\rightleftharpoons}$ aorta between the obstruction and the $\frac{0}{3}$ abdominal measurement position-that is, the same flow passes through the two cross sections of the aorta.

Flow that is accelerated is believed to have a flattened spatial velocity profile. With minor? obstructions the profile is probably skewed. The computer analysis of the flow in the abdominal aorta showed that the spatial velocity profile is not completely flat and thiso is a source of error. The measurement of flow velocity across the lumen too is a source of errors. The measurements are not corrected $\bar{N}_{\mathrm{N}}$ for the sweep velocity of the ultrasonic beam. which skews the profile somewhat. The sweep angle across the abdominal aorta is small and we believe this error is of minor importance Also, if the pulsed Doppler sampling volumeo is positioned in the dilated area in patients ${ }_{\infty}^{\mathbb{D}}$ with post-stenotic dilatation of the abdominal aorta a low velocity will be recorded and afalse diagnosis of severe coarctation may be $\overrightarrow{\mathrm{w}}$ made. We believe the method should be used in older children and adults.

Though there was a good correlation between the velocity ratio and the CoA index, the CoA index was consistently larger. The main reason for this is probably that magnetico resonance imaging gives an anatomical estimation and Doppler gives an estimate of the effective areas. This resembles the use of the continuity equation in valve lesions.

The table shows that the sensitivity of several Doppler variables in detecting a coarcta- 
tion with a CoA index $<0 \cdot 25$ (corresponding to a diameter ratio of 0.5 ) increases when a diastolic half time of more than $100 \mathrm{~ms}$ is included as another criterion. This accords with results obtained by Carvalho et al ${ }^{10}$ in a group of children, many of whom had severe coarctations: our study group consisted predominantly of adults with previous repairs. We believe this difference explains the lower sensitivity/specificity observed in our group when gradients and diastolic half time were used to identify severe coarctations. These older patients probably have more and larger collaterals than in children. If this is the case then the usefulness of gradients is further decreased. ${ }^{12}$

The velocity ratio approach has several practical and theoretical advantages. The uncertainty about use of peak instantaneous gradients on peak to peak gradients is avoided. Likewise the discussion whether or not proximal velocities should be subtracted in the calculation of the Doppler gradient does not apply to our method. Most importantly we believe that whether or not the obstruction is circular the velocity ratio should give adequate information on the cross sectional area. The velocity is a function of the pressure drop across the obstruction and not of the anatomical geometry. In this respect the velocity ratio might be superior to invasive and non-invasive reference methods.

We have confirmed that the correlation between anatomical indices of obstruction and gradients is disappointing. The described velocity ratio method does correlate closely with the anatomical method. The recordings are easy to perform and with this Doppler method it is not necessary to transform the directly measured velocities into gradients.

1 Michaelsson $M$, Sunnegårdh $J$, Hallberg $M$, Áberg $T$. Cuff pressures and Doppler gradients after coarctectomy. A long-term follow-up. Acta Paediatr Scand 1986;Suppl 329:94-7.

2 Presbitero P, DeMarie D, Villani M, Perinetto EA, Riva G, Orzan F, Bobbio M, Morea M, Brusca A. Long term results (15-30 years) of surgical repair of aortic coarcresults $(15-30$ years) of surgical
tation. Br Heart $\mathcal{f} 1987 ; 57: 462-7$.

3 Danielsen R, Nordrehaug JE, Stangeland L, Vik-Mo H. Limitations in assessing the severity of aortic stenosis by Doppler gradients. Br Heart f 1988;59:551-5.

4 Teien D, Human DG, Nanton MA, Wendel H. Evaluation of aortic stenosis by ultrasonographically measured gradients and valve areas in 100 children. $\mathcal{f}$ Cardiovasc Technol 1989;8:273-7.

5 Karp K, Teien D, Bierle P, Eriksson P. Measurement of valve area determinations in mitral stenosis by the pressure half-time method: Impact of left ventricular stiffness and peak diastolic pressure difference. $\exists \mathrm{Am} \mathrm{Coll}$ ness and peak diastolic
Cardiol
9 $13: 594-9$

6 Hanson E, Eriksson BO, Sörensen Sl: Intra-arterial blood pressure at rest and during exeruse after surgery for coarctation of the aorta. Eur f Card:, 1 1989;11:245-57.

7 Teien D, Wendel H, Holm S, Hallbcrg M. Estimation of Doppler gradients at rest and during exercise in patients with recoarctation of the aorta. Br Heart $\mathcal{f}$ 1991;65: $155-7$.

8 Fredriksen T. Coarctation of the aorta. A follow-up examination of an operated material. Scand $\mathcal{f}$ Thorac Cardiovasc Surg 1973; 7: Suppl

9 Hatle L, Angelsen B. Physics of blood flow. In: Doppler ultrasound in cardiology. 2nd ed. Philadelphia: Lea \& ultrasound in cardiology. 2nd
Febiger, 1985: pp 23 and 219.

10 Carvalho JS, Redington AN, Shinebourne EA, Rigby ML, Gibson D. Continuous wave Doppler echocardiography and coarctation of the aorta: gradients and flow patterns in the assessment of severity. Br Heart $\mathcal{F}$ 1990;64:133-7.

11 Bland JM, Altman DG. Statistical methods for assessing agreement between two methods of clinical measurements. Lancet 1986;i:307-10.

12 Wendel H, Teien D, Human DG, Nanton MA. Doppler echocardiographic and morphologic evaluation of patients following operative repair of aortic coarctation. Acta Paediatr Scand 1992;81:247-52. 